\title{
High-mass X-ray binaries in the Magellanic Clouds ${ }^{\star}$
}

\author{
Q. Z. Liu ${ }^{1,2}$, J. van Paradijs² ${ }^{2}$ and E. P. J. van den Heuvel $^{2}$ \\ 1 Purple Mountain Observatory, Chinese Academy of Sciences, Nanjing 210008, PR China \\ e-mail: qzliu@pmo.ac.cn \\ 2 Astronomical Institute, University of Amsterdam, Kruislaan 403, 1098 SJ Amsterdam, The Netherlands
}

Received 29 June 2005 / Accepted 11 July 2005

ABSTRACT

We present a catalogue of high-mass X-ray binaries in the Magellanic Clouds. The catalogue lists source name(s), coordinates, apparent magnitudes, orbital parameters, and X-ray luminosity of 128 high-mass X-ray binaries, together with stellar parameters of the components, other characteristic properties and a comprehensive selection of the relevant literature. The aim of this catalogue is to provide easy access to the basic information on the X-ray sources and their counterparts in other wavelength ranges (UV, optical, IR, radio). Most of the sources are identified to be $\mathrm{Be} / \mathrm{X}$-ray binaries. Some sources, however, are only tentatively identified as high-mass X-ray binaries on the basis of a transient character and/or a hard X-ray spectrum. Further identification in other wavelength bands is needed to finally determine the nature of these sources. In cases where there is some doubt about the high-mass nature of the X-ray binary this is mentioned. Literature published before 1 May 2005 has, as far as possible, been taken into account.

Key words. stars: early-type - X-rays: binaries - Magellanic Clouds - catalogs

\section{Introduction}

High-mass X-ray binaries (HMXBs) were among the very first X-ray sources detected and optically identified more than 3 decades ago. These systems consist of a compact object, mostly a magnetized neutron star (NS) (X-ray pulsar) or a black hole $(\mathrm{BH})$, orbiting a massive star. The X-ray emission in these sources is due to accretion of matter from the early-type masslosing star by the compact companion. Conventionally HMXBs can be further divided into two subgroups (van Paradijs 1983): those in which the primary is a Be star (Be/X-ray binary) and those in which the primary is a supergiant (SG/X-ray binary).

The majority of the known high-mass $\mathrm{X}$-ray binaries are $\mathrm{Be} / \mathrm{X}$-ray systems (BeXRBs). Most Be/X-ray binaries have relatively wide orbits with moderate eccentricity and their compact companions spend most of their time far away from the disc surrounding the Be stars (Coe 2000; Popov \& Raguzova 2005). X-ray outbursts will be expected during the time of the neutron star's periastron passage, in a low-velocity and highdensity wind around Be stars, and thus collectively termed $\mathrm{Be} / \mathrm{X}$-ray transients. Their X-ray spectra are usually hard. The hard X-ray spectrum along with the transience is an important characteristic of the Be/X-ray binaries. In the second group of HMXB systems the compact star orbits a supergiant early-type star, deep inside the highly supersonic wind. The X-ray luminosity is either powered by pure stellar wind accretion or, in

* Tables 1 and 2 are only available in electronic form at the CDS via anonymous ftp to cdsarc.u-strasbg.fr $(130.79 .128 .5)$ or via http://cdsweb.u-strasbg.fr/cgi-bin/qcat? J/A+A/442/1135 the case of the brighter systems, by Roche-lobe overflow via an accretion disk.

The Magellanic Clouds (MCs), satellites of our Galaxy, are the nearest external galaxies. The advantages of studying the X-ray source content of the MCs are well known: they have a relatively small angular size, are at a reasonably close and known distances (conventionally, $65 \mathrm{kpc}$ for the SMC and $55 \mathrm{kpc}$ for the LMC), and are located at a high Galactic latitude with little obscuration by the interstellar medium of the Galaxy. In addition, there is evidence for recent star formation in the MCs, which creates an environment in which HMXBs are expected to be plentiful (Haberl \& Sasaki 2000; Maragoudaki et al. 2001). This expectation is borne out by recent discoveries of a huge number of HMXBs in the MCs based on observations from recent X-ray satellites. Recent reviews of these discoveries are presented in Yokogawa et al. (2003) and Haberl $\&$ Pietsch (2004).

After the first observation of X-ray emission from the MCs in 1968 (Mark et al. 1969), more than 100 HMXBs were found in several satellite missions. Before 1995, the number of known HMXBs in the MCs was about 10 (van Paradijs 1983, 1995). The thorough survey of the MCs made by ROSAT and subsequently systematic study of ROSAT X-ray sources in X-ray and optical bands (Schmidtke et al. 1994; Kahabka \& Pietsch 1996; Cowley et al. 1997; Stevens et al. 1999; Haberl \& Pietsch 1999; Haberl et al. 2000; Coe \& Orosz 2000; Coe et al. 2000) increased the number dramatically. By the time of the previous catalogue the number of X-ray sources associated with massive stars in the MCs had increased to 45 (Liu et al. 2000). 
The amount of new literature and the number of HMXB in the MCs have grown so much that it is now useful to concentrate the information of HMXBs in the MCs in a separate catalogue. In this paper we present a catalogue of $128 \mathrm{HMXBs}$ in the MCs reported in the literature up to 1 May 2005. In further works, we will present the catalogues of HMXBs and LMXBs in the Galaxy.

\section{Description of the table}

Tables 1 and 2 list the 92 HMXBs in the SMC and 36 HMXBs in the LMC, respectively. The format of the tables is similar to that of the previous ones (Liu et al. 2000, 2001), of which the present catalogue is meant to be an update. In the table the sources are ordered according to right ascension of sources; part of the (mainly numerical) information on a source is arranged in six columns, below which for each source additional information is provided in the form of key words with reference numbers [in square brackets]. The columns have been arranged as follows.

In Col. 1 the first line contains the source name, with rough information on its sky location, according to the conventional source nomenclature of space satellites in which the source was detected, hhmm \pm ddd, or hhmm.m \pm ddmm. Here hh, $\mathrm{mm}$ and ss indicate the hours, minutes and seconds of right ascension, ddd the declination in units of 0.1 degree (in a small number of cases, the coordinates shown in the name are given with more, or fewer, digits). The prefix $\mathrm{J}$ indicates a name based on J2000 coordinates. Otherwise, 1950 coordinates were used in the name. An alternative source name is given in the second line. In the third line of Col. 1, the source types are indicated with a letter code, as follows:

- P: X-ray pulsar (47 in the SMC and 7 in the LMC);

- T: transient X-ray source;

- U: ultra-soft X-ray spectrum. These sources include blackhole candidates; some "extreme ultra-soft" (EUS) source may be white dwarf (WD) on whose surface steady nuclear burning takes place.

Column 2 contains in the first two lines the right ascension (RA) and declination (DEC) of the source for epoch 2000. RA is given as hhmmss.s to an accuracy of $0.1 \mathrm{~s}$, Dec is given in ${ }^{\circ} \prime \prime$, to an accuracy of $1^{\prime \prime}$ (in a small number of cases, the coordinates are given with more, or fewer, digits). The third line gives the galactic longitude and latitude to an accuracy of $0.1^{\circ}$. A reference on the source position is given below the columnar information under "pos.". In the parentheses following the "pos." we provide some information on the type of observation from which the source position has been derived. The following abbreviations have been used: o, optical; $x$, X-ray; r, radio; IR, infrared. Following the type of observation, we give an indication of the accuracy of this position, in the form of equivalent (90 percent confidence level) error radii, but in several cases this can only be considered an approximation (e.g. when the error box is not circular). When no accuracy is quoted, it is about one arcsecond or better.

The first and second lines of Col. 3 give names of an optical counterpart. The third line contains a reference to a finding chart. An asterisk followed by a number or letter refers to star numbers used in the finding chart; "star" refers a star in the finding chart that has not been assigned a number or letter. Many optical counterparts have been indicated with a $\mathrm{H} \alpha$ emission-line object in the SMC by Meyssonier \& Azzopardi (1993, hereafter MA93) and a Small Magellanic Cloud member star by Azzopardi \& Vigneau (1982, hereafter $\mathrm{AzV}$ ), or a number in a well-known catalogue (e.g., GSC, 2MASS, OGLE, MACHO etc.).

The fourth column contains some photometric information on the optical counterpart. In the first line, the apparent visual magnitude, $V$, and the color indices $B-V$, and $U-B$, are listed. The second line contains the spectral type of the optical counterpart and an estimate of the interstellar reddening, $E_{B-V}$.

In Col. 5, the average X-ray flux, or the range of observed $\mathrm{X}$-ray fluxes (2-10 keV, unless otherwise indicated), is given, in units of

$$
\begin{aligned}
1 \mu \mathrm{Jy} & =10^{-29} \mathrm{erg} \mathrm{cm}^{-2} \mathrm{~s}^{-1} \mathrm{~Hz}^{-1} \\
& =2.4 \times 10^{-12} \mathrm{erg} \mathrm{cm}^{-2} \mathrm{~s}^{-1} \mathrm{keV}^{-1}
\end{aligned}
$$

The first line in Col. 6 gives the orbital period in days. The second line contains for X-ray pulsars the pulse period, in seconds. The third line contains references in which the orbital and/or pulse periods were detected.

\section{Remarks on some individual objects}

We wish to emphasize that the nature of some sources listed in this catalogue is still uncertain. They are tentatively classified as massive X-ray binaries due to their X-ray transience and/or hard X-ray spectra. No counterpart at other bands has been found or no pulse period has been detected, or both. Also, the compact object in some weak or soft X-ray sources may be a white dwarf instead of a neutron star. If this is the case, they should be excluded from this catalogue.

During a Chandra observation at the NGC 346 field in the SMC, a few dozens of new X-ray sources were discovered (Naze et al. 2003). 32 X-ray sources were found to have possible counterparts, in which a large number of these counterparts were estimated to be a B spectral type star, suggested that most of these X-ray sources are in fact X-ray binaries (Naze et al. 2003). RX J0104.7-7203 (source No. 186, an AGN candidate, in Kahabka et al. 1999) was clearly missing (Naze et al. 2003). Therefore, this source is likely a transient X-ray binary. Two other ROSAT sources (Nos. 61 and 75 in Sasaki et al. 2000) should be present in the Chandra field but were not clearly detected (Naze et al. 2003), probably also transient sources. However, we have only included one of the sources in our catalogue (source No. 68 in Naze et al. 2003), because its hard X-ray spectrum, as well as the spectral type and optical colors, is consistent with HMXB nature.

In addition, we would like to make some remarks on additional 2 pulsar sources in the SMC. We have not included them in this catalogue, because of the uncertainty of the nature of the two sources. The $0.088 \mathrm{~s}$ pulsar, AX J0043-737, is a Crab-like pulsar in the SMC with X-ray luminosity of $8.6 \times 10^{34} \mathrm{erg} \mathrm{s}^{-1}$ (Yokogawa \& Koyama 2000; 
Yokogawa et al. 2003). Its nature is uncertain, although the possibility of an X-ray binary with a short pulse period is not excluded (Yokogawa \& Koyama 2000). The 8.02 s X-ray pulsar, CXOU J010043.1-721134, was classified as a possible anomalous X-ray pulsar (AXP) (Lamb et al. 2002; McGarry et al. 2005) from its $X$-ray properties, although a B3V star with $V=17.7, B-V=-0.03$, and $U-B=-0.68$ is found within the error of the X-ray source and its spectral type and optical colors are consistent with HMXB nature (Naze et al. 2003; Shtykovskiy \& Gilfanov 2005b).

Finally, there are another 63 weak hard X-ray sources in the SMC (Kahabka et al. 1999; Sasaki et al. 2000) and 9 at the northern area of the supergiant shell LMC 4 (Kahabka 2002). However, it is not clear if they are X-ray binaries or background AGNs according to the hard X-ray spectra (Kahabka et al. 1999). Also there are some X-ray binary candidates in the MCs detected with XMM (Shtykovskiy \& Gilfanov 2005a,b). In view of the uncertainty of the nature of these sources, they are not included in this catalogue.

\section{Discussion}

\subsection{HMXB populations in the MCs}

$\mathrm{X}$-ray satellite observations have revealed that the MCs contain an unexpectedly large number of high-mass X-ray binaries, especially in the Small Magellanic Cloud. Up to now, 92 known or probable HMXB sources in the SMC and 36 HMXBs in the LMC have been identified and they continue to be discovered, although only a small fraction of these are active at any one time because of their transient nature. Of the $128 \mathrm{HMXBs}$ in this catalogue, 4 of the objects are supergiant systems (EXO 0114.6-7361, SMC X-1, RX J0532.4-6535, and RX J0541.4-6936), and all the rest are likely Be/X-ray binaries. To compare the HMXB populations in the MCs and in our Galaxy, we collected the HMXBs in the three galaxies from our previous X-ray binary catalogues (van Paradijs 1983, 1995; Liu et al. 2000) and recent literature. Figure 1 shows the numbers of known HMXB candidates in the three galaxies. The numbers of HMXBs in the LMC and the Galaxy increase gradually while the number in the SMC shows a steep upward trend without sign of levelling.

Table 3 summarizes the X-ray binary populations in the three galaxies, where the numbers of sources are normalized by the mass ratio of the galaxies (our Galaxy : LMC : SMC $\approx$ $100: 10: 1$ ) for a simple comparison. The LMXBs are collected from the X-ray catalogue (Liu et al. 2001, and the update version, 2005). Apparently the normalized number of HMXBs is much higher in the SMC than in our Galaxy and the LMC. Unusually, all but one of the X-ray binaries so far discovered in the MCs are HMXBs. The number ratio of HMXBs to LMXBs exhibits a striking difference between the MCs and our Galaxy, as is already pointed out by Schmidtke et al. (1999) and Yokogawa et al. (2003). The number in this catalogue makes the difference larger. Naze et al. (2003) points out that there may be hundreds more. Such a high density of HMXB population must provide clues about the star formation rates in the SMC, since HMXBs could be an indicator of star formation

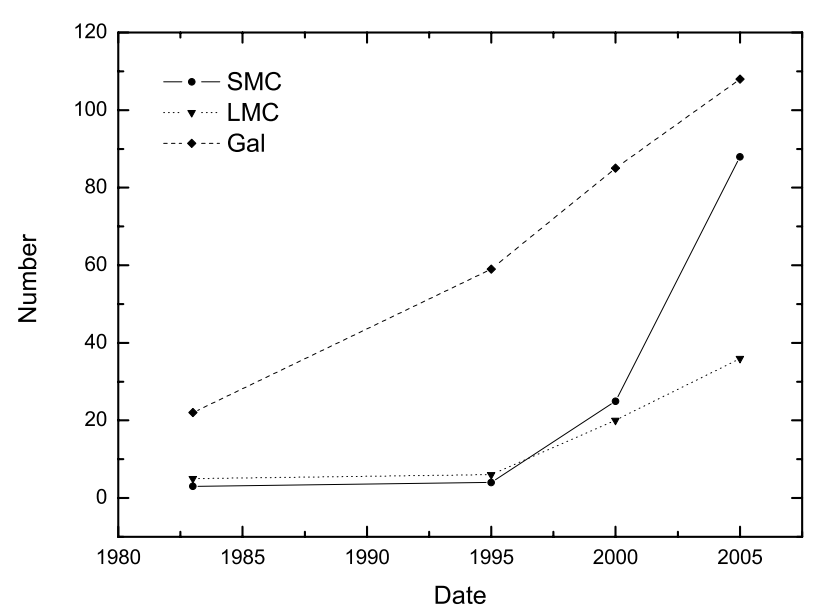

Fig. 1. Number of high-mass X-ray binaries in the SMC, the LMC and the Galaxy.

Table 3. Source populations in the MCs and in the Galaxy.

\begin{tabular}{lccccc}
\hline \hline Galaxy & \multicolumn{3}{c}{ HMXBs } & \multicolumn{2}{c}{ LMXBs } \\
\hline & Total & Pulsar & BHC & Total & BHC \\
\hline SMC & 92 & 47 & 0 & 0 & 0 \\
SMC $* 100$ & 9200 & 4700 & 0 & 0 & 0 \\
LMC & 36 & 7 & 2 & 1 & 0 \\
LMC $* 10$ & 360 & 70 & 20 & 10 & 0 \\
Galaxy & 108 & 57 & $1:$ & 178 & $\geq 39$ \\
\hline
\end{tabular}

Note: Data are from this catalogue and the updated catalogues of Liu et al. $(2000,2001)$.

rate (Grimm et al. 2003) while LMXBs are generally considered to comprise an older population than HMXBs. The MCs should be much active in massive star formation, which is consistent with the results that MCs have experienced encounters with the Galaxy in past and the encounters likely triggered bursts of star formation (see e.g. Yoshizawa \& Noguchi 2003).

In contrast to the large number of HMXBs in the MCs, it is very unusual that there are no black hole X-ray binaries in the SMC and only two black hole candidates in the LMC, while more than 40 black hole candidates were found in the Galaxy. However, Zhang et al. (2004) found that it is very difficult to detect black hole/Be systems, due to the efficient truncation of the Be circumstellar disk by black holes.

\subsection{Pulse period distribution of HMXBs}

A simple scaling based on the relative masses of the MCs and the Galaxy predicts only 2 pulsars in the SMC and 20 in the LMC. However, currently 49 X-ray pulsars are known in the SMC (Coe et al. 2005; Haberl \& Pietsch 2005), and 7 in the LMC. All pulsars except 2 in the SMC are most likely in HMXB systems as suggested by their X-ray properties (Haberl $\&$ Pietsch 2004). Figure 2 shows the pulse period distributions of the X-ray binary pulsars in the MCs and our Galaxy. In average, the pulse periods in the SMC are slightly shorter than 


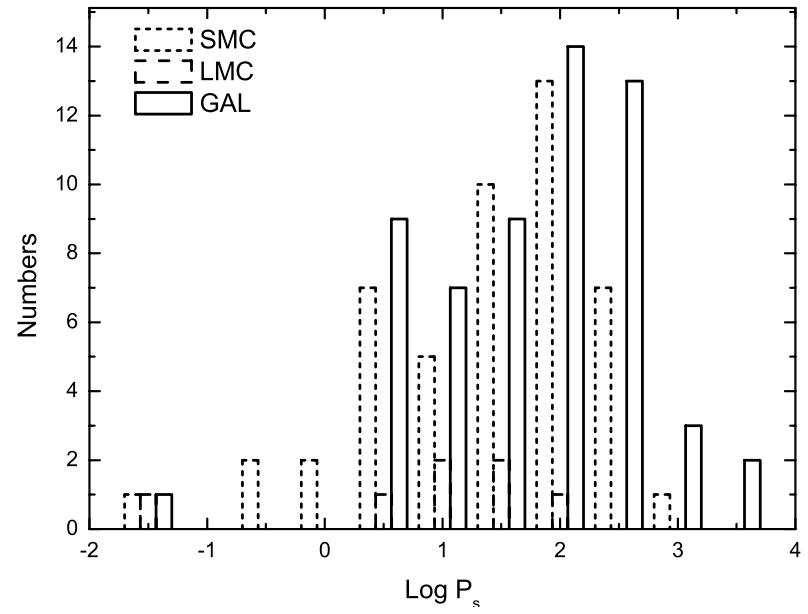

Fig. 2. A comparison of the pulse period distributions of the X-ray pulsars in the SMC, the LMC and the Galaxy.

those in the Galaxy, but not very apparent. Since the pulsars in the SMC are likely much younger than those in the Galaxy, we should see the reversal if the pulsars in Be/X-ray binaries are really spin-up.

Acknowledgements. We are very grateful to the referee, Malcolm Coe for his carefully reading the manuscript and useful comments. This research has made use of the SIMBAD data base, operated at CDS, Strasbourg, France, and NASA's Astrophysics Data System (ADS). Q.Z.L. acknowledges support from the Major State Basic Research Development Program of China (973 Program) under Grant G1999075405 and the National Natural Science Foundation of China under Grants Nos. 10173026 and 10433030.

\section{References}

Azzopardi, M., \& Vigneau, J. 1982, A\&AS, 50, 291

Coe, M. J. 2000, in The Be Phenomenon in Early-Type Stars, ed. M. A. Smith, \& H. F. Henrichs (San Francisco: ASP), IAU Colloq., 175, 656

Coe, M. J., \& Orosz, J. A. 2000, MNRAS, 311, 169

Cowley, A. P., Schmidtke, P. C., McGrath, T. K., et al. 1997, PASP, 109,21
Grimm, H.-J., Gilfanov, M., \& Sunyaev, R. 2003, MNRAS, 339, 793

Haberl, F., \& Pietsch, W. 1999, A\&AS, 139, 277

Haberl, F., \& Pietsch, W. 2005, A\&A, 438, 211

Haberl, F., \& Sasaki, M. 2000, A\&A, 359, 573

Haberl, F., Filipovic, M. D., Pietsch, W., \& Kahabka, P. 2000, A\&AS, 142,41

Haberl, F., Pietsch, W., Schartel, N., et al. 2004, A\&A, 420, L19

Kahabka, P. 2002, A\&A, 388, 100

Kahabka, P., \& Pietsch, W. 1996, A\&A, 312, 919

Kahabka, P., Pietsch, W., Filipovic, M. D., \& Haberl, F. 1999, A\&AS, 136,81

Lamb, R. C., Macomb, D. J., Prince, T. A., \& Majid, W. A. 2002, ApJ, 567, L129

Liu, Q. Z., van Paradijs, J., \& van den Heuvel, E. P. J. 2000, A\&AS, 147,25

Liu, Q. Z., van Paradijs, J., \& van den Heuvel, E. P. J. 2001, A\&A, 368,1021

Maragoudaki, F., Kontizas, M., Morgan, D. H., et al. 2001, A\&A, 379, 864

Mark, H., Price, R., Rodrigues, R., et al. 1969, ApJ, 155, L143

McGarry, M. B., Gaensler, B. M., Ransom, S. M., et al. 2005, ApJ, 627, L137

Meyssonnier, N., \& Azzopardi, M. 1993, A\&AS, 102, 451 (MA93)

Naze, Y., Hartwell, J. M., Stevens, I. R., et al. 2003, ApJ, 586, 983

Popov, S. B., \& Raguzova, N. V. 2005, A\&A [arXiv: astro-ph/0405633]

Sasaki, M., Haberl, F., \& Pietsch, W. 2000, A\&AS, 147, 75

Schmidtke, P. C., Cowley, A. P., Frattare, L. M., et al. 1994, PASP, 106,843

Schmidtke, P. C., Cowley, A. P., Crane, J. D., et al. 1999, AJ, 117, 927

Shtykovskiy, P., \& Gilfanov, M. 2005a, A\&A, 431, 597

Shtykovskiy, P., \& Gilfanov, M. 2005b, MNRAS [arXiv: astro-ph/0503477]

Stevens, J. B., Coe, M. J., \& Buckley, D. A. H. 1999, MNRAS, 309, 421

Van Paradijs, J. 1983, in Accretion Driven Stellar X-ray Sources, ed. W. H. G. Lewin, \& E. P. J. van den Heuvel, Cambridge, 189

Van Paradijs, J. 1995, in X-ray Binaries, ed. W. H. G. Lewin, J. Van Paradijs, \& E. P. J. van den Heuvel, Cambridge, 536

Yokogawa, J., \& Koyama, K. 2000, IAUC, 7361

Yokogawa, J., Imanishi, K., Tsujimoto, M., et al. 2003, PASJ, 55, 161

Yoshizawa, A. M., \& Noguchi, M. 2003, MNRAS, 339, 1135

Zhang, F., Li, X.-D., \& Wang, Z.-R. 2004, ApJ, 603, 663 